\title{
НАПРАВЛЕНИЯ И СПОСОБЫ ВОЗДЕЙСТВИЯ ГЛОБАЛИЗАЦИИ НА СИСТЕМУ РЕГУЛИРОВАНИЯ ИНТЕЛЛЕКТУАЛЬНЫХ ПРАВ: ТЕОРЕТИЧЕСКИЕ АСПЕКТЫ
}

\begin{abstract}
Аннотация: В эпоху глобализации основой экономии являются информация и знания, что определяет растущее значение регулирования интеллектуальных прав. В статье анализируются различные подходы коценке роли права интеллектуальной собственности в стимулировании инновационного развития экономики, которые свидетельствуют о неоднозначности тезиса о необходимости усиления защить правообладателей. Унификация международного законодательства в сфере интеллектуальной собственности, как показывает автор, не учитывает особенностей экономического развития различных государств, и может привести к негативным последствиям. Гибкий и дифференцированный подход крегулированию интеллектуальных прав в большей степени соответствует характеруизменений, которое претерпеваетправо под воздействиемглобализации. Особое вниманиев статьеуделяется проблеме соотношения интеллектуальньх прав и прав человека, которая в условиях глобализации становится крайне актуальной. В заключение автором анализируется несоответствие между общили тенденцияли развития права в эпоху глобализации и основныли направленияли развития системы регулирования интеллектуальных прав, что позволяет сделать вывод о кризисе действующей парадигмы интеллектуальных прав.

Abstract: In the globalization era information and knowledge represent the cornerstone of economy, which defines the growing significance of regulating the intellectual rights. This article analyzes various approaches towards assessment of the role of intellectual property law in stimulation of the innovational development of economy, which testify to the ambiguity of the argument about the need to strengthen the protection of the right holders. The author demonstrates that the unification of international legislation in the area of intellectual property does not account for the specifics of the economic development of different countries and can lead to negative consequences. A flexible and differentiated approach towards the regulation of intellectual rights mostly corresponds to the nature of changes to the law under the effects of the globalization process. A special attention is given to the problem of correlation between intellectual rights and human rights, which under the conditions of globalization becomes extremely relevant. In the conclusion, the author analyzes the inconsistencies between the general tendencies of development of law in the era of globalization and the main trends of development within the regulation system of intellectual right, which allows us to come to a conclusion on the crisis of the current paradigm of intellectual rights.

Ключевые слова: Глобализация, интеллектуальные права, теория права, права человека, правовая парадигма, экономика знания, информация, информационные технологии, общественное благо, гармонизация законодательства.
\end{abstract}

Keywords: Globalization, intellectual rights, legal theory, human rights, legal paradigm, economy of knowledge, information, information technologies, public benefit, harmonization of legislation.

лобализация и информационные технологии теснейшим образом взаимосвязаны, поэтому правовой ландшафт сегодня определяется не только глобализацией как интенсификаций взаимодействия в различных сферах и на различных уровнях, но и собственно информационными технологиями, т.е. тем способом, которым осуществляются коммуникационные процессы1. Поскольку глобализация связана с определенным типом экономики, а именно - экономикой знания, важнейшую роль в современном праве играет система регулирования интеллектуальных прав, которая устанавливает основные правила, касающиеся защиты, доступа и распространения технологий и инноваций.

Исследуя интеллектуальную собственность как составную часть глобализации лауреат нобелевской премии в области экономики Джозеф Штиглиц убедительно доказывает ошибочность общепринятого тезиса о том, что основная функция интеллектуальных прав заключается в стимулировании экономического роста. Как полагает ученый, несбалансированные правовые режимы в сфере интеллектуальной собственности «приводят к менее эффективной экономике и могут даже замедлить темпы развития инноваций»². Позиция,

\footnotetext{
${ }^{1}$ См. Например: Marsden C. T. Introduction. Information and communications technologies, globalisation and regulation. // Regulating the global information society. Edited by Christopher T.Marsden. Routledge, 2000. P. $1-40$.
}

${ }^{2}$ Stiglitz J.E. Making globalization work. Norton \& Company, 2006. P. 106. согласно которой инновационное развитие можно обеспечить исключительно с помощью предоставления монопольного права, непосредственно корреспондирует «рыночный фундаментализм», т.е. убеждение в том, что рынок сам по себе приводит к экономической эффективности. Работы Дж. Штиглица по экономике публичного сектора показывают необходимость нахождения баланса между свободным рынком и государственным управлением. Точно также и право интеллектуальной собственности, если его действительной целью является поддержка инноваций и защита общественного блага, должно опираться на баланс интересов авторов, корпораций, ученых, потребителей, общества и государства ${ }^{3}$.

Изучение системы регулирования интеллектуальных прав в контексте глобализации связано, в первую очередь, с темой информации и информационных потоков. Новое качество коммуникаций и доступа к данным, которые обеспечивают информационные технологии, специалисты рассматривают как феномен «глобализации информации» ${ }^{4}$ Масштабы информационного обмена и значение информации для экономического развития предполагают новую «систему координат»: интеллектуальные права рассматриваются
${ }^{3}$ Stiglitz J.E. Making globalization work. Norton \& Company, 2006. P.XIII, 106, 108, 112-115.

${ }^{4}$ См., например: Cate F. H. Introduction: Sovereignty and the Globalization of Intellectual Property, Symposium. // Indiana Journal of Global Legal Studies. 1998. Vol. 6. Issue 1. P.1-3. 
уже не как самостоятельная область правового регулирования, а как часть общей системы регулирования информационной сферы. Доступность информации (с точки зрения стоимости, скорости и простоты копирования) и ее ключевая роль в современной экономики (одновременно и как технологии производства и организации бизнес-процессов, и как востребованного товара и услуг) актуализировали вопрос о том, кто и на каких условиях владеет информацией, а также обусловили формирование целого комплекса, подходов, позиций и стратегий.

Правообладатели и те, кто строили свою коммерческую деятельность по традиционным бизнес-моделям, отстаивают усиление правовой защиты интеллектуальных прав как единственного сдерживающего фактора для взросшего числа правонарушений. Согласно такому подходу, уменьшение правовой защиты приведет к резкому снижению как инноваций в сфере технологий и промышленности, так и творческой активности в сфере науки и культуры. Информационные посредники, основная деятельность которых непосредственно связана с цифровой средой, напротив, поддерживают идею об упрощении правового режима интеллектуальных прав, сокращению сроков правовой защиты, легализации оцифровки произведений без согласия их авторов и т.п. Сходную позицию занимает академическое сообщество, поскольку результаты их научных и творчески поисков во все исторические периоды, но в особенности в цифровую эпоху, зависят от осведомленности о результатах деятельности и от коммуникации со своими коллегами, т.е. от максимально открытого доступа к информации. С другой стороны, преодоление цифрового неравенства, как составная часть глобальной политики в сфере телекоммуникаций, привело к тому, что доступ к Интернету и информационным ресурсам стал доступным для миллионов людей. Цифровые технологии стали важным элементом системы образования, резко возрос объем произведений со свободным доступом, создателями которых выступают не профессионалы, а сами пользователи Интернета. Эти и другие феномены обусловили формирование в обществе новой идеологии, в соответствии с которой свободный доступ к информации и знаниям является общественным благом и правом человека, а защита правообладателей служит в первую очередь интересам крупных корпораций.

Другой вопрос, который широко обсуждается в связи с воздействием глобализации на право интеллектуальной собственности, заключается в гармонизации национального и международного законодательства в данной сфере. Соглашение по торговым аспектам прав интеллектуальной собственности (ТРИПС) не только занимает важное место в системе ВТО, но представляет собой также наиболее значимую инициативу по гармонизации системы защиты интеллектуальных прав в глобальном масштабе. Международным актом устанавливаются стандарты и комплекс минимальных требований в сфере защиты патентов, авторского права, товарных знаков и других объектов интеллектуальной собственности. Практически сразу после своего принятия соглашение стало предметом дискуссий как в научной среде (прежде всего, среди экономистов, политологов и юристов), так за ее пределами с активным участием неправительственных организаций и глобальных движений.

Основная проблема, которая явным образом обозначили многочисленные споры, заключается в правомерности установления единых и системных правовых требований в такой сфере регулирования как право интеллектуальной собственности. В научной литературе противостояние двух позиций, обычно, ассоциируются с двумя противоположными тезисами - «один размер подходит для всех» и «один размер не подходит для всех»5. В условиях интенсификации межнациональных экономических и культурных взаимодействий очевидна потребность в унификации правовых условий в целях повышения правовой определенности. С другой стороны, высокий уровень унифицированных требований может не соответствовать особенностям экономического развития различных государств (уровню, структуре и институциональной составляющей национальной экономики).

Хотя ни ученые, ни политики до настоящего времени не пришли к общему мнению по данному вопросу, представляется, что гибкий и дифференцированный подход к регулированию интеллектуальных прав в большей степени соответствует характеру изменений, которое претерпевает право под воздействием глобализации. Мы солидарны с позицией, высказанной по данному вопросу Комитетом по экономическим, социальным и культурным правам ООН. Согласно мнению Комитета, страны различаются как по уровню своего экономического развития, так и по потребностям в развитии и использовании технологий. Соответственно, «международные правила, касающиеся интеллектуальной собственности, не должны быть с необходимостью унифицированными, если это может привести к тому, что защита интеллектуальной собственности будет не соответствовать целям устойчивого развития» ${ }^{6}$.

Спорность вопроса об унификации международного регулирования интеллектуальной собственности лишний раз подтверждает, насколько противоречивым является феномен глобализации. Так, доктор экономических наук, профессор, В.С. Паньков приходит к выводу, что глобализация, с одной стороны, создает необходимые предпосылки для повышения эффективности мирового хозяйства и социального прогресса, a, с другой стороны, реализуется в таких формах, которые приводят к ущемлению интересов широких слоев населения и целых стран, «не входящих в известный «клуб» развитых государств «золотого миллиарда».

В связи с развитием в условиях глобализации «тенденции расширения и усиления законодательства, касающегося прав и свобод человека и гражданина» ${ }^{8}$, необходимо упомянуть также проблему соотношения интеллектуальных прав и

${ }^{5}$ См., например: Barton J. H., Abbott F. M., Correa C. M., Drexl J., Foray D., Marchant $R$. Views on the Future of the Intellectual Property System. ICTSD Programme on Intellectual Property Rights and Sustainable Development. Geneva: ICTSD, 2007; Structure of Intellectual Property Law: Can One Size Fit All? Ed. by Kur A., Mizaras V. ATRIP. Edward Elgar Pub, 2011; Carroll M. W. One Size Does Not Fit All: A Framework for Tailoring Intellectual Property Rights. // Ohio State Law Journal. Vol. 70. № 6. 2009. P.1361-1434.

${ }^{6}$ Human rights and intellectual property. Statement of Committee on Economic, Social and Cultural Rights. United Nations. Document № C.12/2001/15. P.5.

7 Паньков В.С. Глобализация экономики: некоторые дискуссионные вопросы. //Золотой лев. 2007. №142. С.40.

${ }^{8}$ Марченко М. Н. Государство и право в условиях глобализации. М.: Проспект, 2009. С.290. 
DOI: $10.7256 / 1811-9018.2014 .12 .13718$

При цитировании этой статьи сноска на dоі обязательна

\section{Право и политика $12(180) \cdot 2014$}

прав человека. Помимо значительного количества научной литературы по данной тематике 9 , о фундаментальном характере проблемы свидетельствует активное обсуждение проблемы в Организации объединенных наций $(\mathrm{OOH})^{10}$. В центре внимания ООН оказались как вопросы оценки обеспечения прав человека при толковании и применении положений ТРИПС, так и общий концептуальный подход. Согласно позиции Комитета $\mathrm{OOH}$ по экономическим, социальным и культурным правам, экономическая деятельность, включая торговлю, финансы и инвестиции, не обладает каким-либо особым статусом, а значит, права человека должны соблюдаться здесь так же, как и во всех других сферах общественной жизни ${ }^{11}$.

Исходя из проведенного анализа, мы можем сделать ряд выводов, как содержательного, так теоретико-методологического характера.

$\mathrm{C}$ точки зрения глобальных перспектив развития права интеллектуальной собственности, представляется важным следование определенной целевой установке. В эпоху глобализации человечество обладает всеми необходимыми ресурсами для того чтобы сократить существующие цифровое и инновационное неравенство. Действующая система интеллектуальных прав и соответствующая правовая парадигма, напротив, закрепляют и увеличивают разрыв в развитии коммуникаций и инноваций, который существует сегодня между странами с развитой и развивающейся экономикой.

Поскольку в эпоху глобализации происходит становление информационного общества, вопросы обеспечения граждан доступом к информации и знаниям постепенно выходят за границы политики и идеологии и обретают свою правовую реальность в качестве неотъемлемых прав человека и правовых принципов. Соблюдение указанных прав и принципов становится важным критерием для оценки легитимности и эффективности существующей системы регулирования интеллектуальных прав. Это означает, что основополагающий тезис о необходимости усиления защиты прав авторов и изобретателей в условиях опережающего развития информационных технологий должен быть дополнен необходимостью поиска баланса интересов в целях соблюдения прав потребителей, пользователей и общества в целом.

В заключении важно подчеркнуть, что общие закономерности в развитии и модернизации права, наличие которых подтверждается исследованиями ведущих российских и зарубежных специалистов в области теории и философии права, должны находить свое отражение также и в такой области правового регулирования как право интеллектуаль-

\footnotetext{
${ }^{9}$ См., например: Human rights in the global information society. Ed. by Jørgensen.R.F. MIT Press, 2006; Human Rights and Intellectual Property Rights: Tensions and Convergences. Ed. by: Sinjela M. Leiden: Nijhoff Publishers, 2007; Hefler L.R., Austin G.W. Human rights and intellectual property : mapping the global interface. Cambridge University Press, 2011.

${ }^{10}$ Cм.: The impact of the Agreement on Trade-Related Aspects of Intellectual Property Rights on human rights. Report of the High Commissioner for Human Rights. Economic and Social Council. United Nations. Document № E/CN.4/Sub.2/2001/13; Human rights and intellectual property. Statement of Committee on Economic, Social and Cultural Rights. United Nations. Document № C.12/2001/15.

${ }^{11}$ Cм.: Statement of the Committee on Economic, Social and Cultural Rights to the Third Ministerial Conference of the World Trade Organization. 26 November 1999. United Nations. Document № E/C.12/1999/9.
}

ной собственности. Однако анализ действующей системы регулирования интеллектуальных прав показывает, что данная система развивается преимущественно изолированно и оказывает пассивное или активное сопротивление общеправовым тенденциям.

Bo-nepвыx, глобализация в праве приводит к продуктивному взаимодействию правовых систем и рецепции правовых институтов, которые позволяют национальному праву более эффективно справляться с актуальными практическими проблемами. В области интеллектуальной собственности, напротив, происходит дальнейшее развитие тех идеологических установок и стандартов, которые были сформированы в доцифровую эпоху, и которые препятствуют гармоничному и сбалансированному регулированию информационной сферы в целом. С точки зрения политической экономики, данная тенденция означает правовое закрепление преимуществ и приоритета государств с инновационной экономикой в ущерб тем государствам, в которых инновационные направления развития национальной экономики находятся на этапе своего становления.

Bo-вmopbx, глобализация с ее усложнением структуры общественных отношений и коммуникаций принуждает право к смещению акцентов с законодательного регулирования, которое еще недавно воспринималось как синоним правового регулирования, на использование максимально широкого спектра способов регулирования и привлечение к процессу регулирования различных субъектов. Обозначая данную тенденцию как гибкость регулирования, мы полагаем, что ее развитие является основным средством, с помощью которого право адаптируется к динамично изменяющимся общественным отношениям и получает возможность находить своевременные и адекватные правовые ответы на вызовы глобализации. Оценка права интеллектуальной собственности с точки зрения гибкости регулирования дает отрицательные результаты. Использование в данной сфере норм рекомендательного характера, обращение к механизмам саморегулирования является, скорее, исключением, чем правилом.

$\boldsymbol{B}$-mpembux, увеличение в условиях глобализации роли и значения прав и свобод человека как в международном, так и в национальном праве, не находит должного отражения в существующей системе регулирования интеллектуальных прав. Право интеллектуальной собственности, как и столетие назад, продолжает оставаться правом, защищающим исключительно интересы правообладателей. Существующие в данной системе ограничения в последние десятилетия имеют тенденцию к сокращению, а объем интеллектуальных прав и перечень объектов защиты, напротив, расширяется. Неудивительно, что в отсутствии работающего механизма, обеспечивающего необходимый баланс интересов всех участников, действующая система регулирования не справляется с запросами современности и оказывается неспособной служить целям роста общественного благосостояния и стимулирования развития науки и культуры.

Несоответствие между общеправовыми тенденциями и закономерностями и регулированием интеллектуальных прав, о чем свидетельствуют приведенные выводы, указывает на наличие глубинных проблем и противоречий, которые затрагивают не какие-то отдельные аспекты регулирования или только некоторые объекты интеллектуальных прав, а систему в целом и правовую парадигму, лежащую в ее основе. 
DOI: $10.7256 / 1811-9018.2014 .12 .13718$

При цитировании этой статьи сноска на доі обязательна

Трансформация правовых и политических систем

\section{Библиография:}

1. Марченко М. Н. Государство и право в условиях глобализации. М.: Проспект, 2009. - 400 с.

2. Паньков, В.С. Глобализация экономики: некоторые дискуссионные. //Золотой лев. - 2007. - №142. - С.24-75.

3. Barton J. H., Abbott F. M., Correa C. M., Drexl J., Foray D., Marchant R. Views on the Future of the Intellectual Property System. ICTSD Programme on Intellectual Property Rights and Sustainable Development. Geneva: ICTSD, 2007. - 41 p.

4. Carroll M. W. One Size Does Not Fit All: A Framework for Tailoring Intellectual Property Rights. // Ohio State Law Journal. Vol. 70 . № 6. 2009. P.1361-1434.

5. Cate F. H. Introduction: Sovereignty and the Globalization of Intellectual Property, Symposium. // Indiana Journal of Global Legal Studies. 1998. Vol.

6. Issue 1. P.1-9. 6.Hefler L.R., Austin G.W. Human rights and intellectual property : mapping the global interface. Cambridge University Press, 2011. $-568 \mathrm{p}$.

7. Human rights in the global information society. Ed. by Jørgensen.R.F. MIT Press, 2006. -324 p

8. Human Rights and Intellectual Property Rights: Tensions and Convergences. Ed. by: Sinjela M. Leiden: Nijhoff Publishers, 2007. - 303 p.

9. Marsden C. T. Introduction. Information and communications technologies, globalisation and regulation. // Regulating the global information society. Edited by Christopher T.Marsden. Routledge, 2000. P. 1-40.

10. Stiglitz J.E. Making globalization work. New York: Norton \& Company, 2006. - 358 p

11. Structure of Intellectual Property Law: Can One Size Fit All? Ed. by Kur A., Mizaras V. ATRIP. Edward Elgar Pub, 2011. - 384 p.

\section{References (transliterated):}

1. Marchenko M. N. Gosudarstvo i pravo v usloviyakh globalizatsii. M.: Prospekt, 2009. $-400 \mathrm{~s}$

2. Pan'kov, V.S. Globalizatsiya ekonomiki: nekotorye diskussionnye. //Zolotoi lev. - 2007. - №142. - S.24-75.

3. Barton J. H., Abbott F. M., Correa C. M., Drexl J., Foray D., Marchant R. Views on the Future of the Intellectual Property System. ICTSD Programme on Intellectual Property Rights and Sustainable Development. Geneva: ICTSD, 2007. - 41 p.

4. Carroll M. W. One Size Does Not Fit All: A Framework for Tailoring Intellectual Property Rights. // Ohio State Law Journal. Vol. 70 . № 6. 2009. P.1361-1434.

5. Cate F. H. Introduction: Sovereignty and the Globalization of Intellectual Property, Symposium. // Indiana Journal of Global Legal Studies. 1998. Vol. 6. Issue 1. P.1-9.

6. Hefler L.R., Austin G.W. Human rights and intellectual property : mapping the global interface. Cambridge University Press, $2011 .-568$ p.

7. Marsden C. T. Introduction. Information and communications technologies, globalisation and regulation. // Regulating the global information society. Edited by Christopher T.Marsden. Routledge, 2000. P. 1-40.

8. Stiglitz J.E. Making globalization work. New York: Norton \& Company, 2006. $-358 \mathrm{p}$. 